\title{
Estimation of Longitudinal Tire Force Using Nonlinearity Observer
}

\author{
Suwat Kuntanapreeda \\ Department of Mechanical and Aerospace Engineering, Faculty of Engineering, \\ King Mongkut's University of Technology North Bangkok, Bangkok, Thailand \\ Email: suwat@kmutnb.ac.th
}

Received 2013

\begin{abstract}
Tire forces are the major forces propelling the road vehicles. They significantly affect the dynamic behavior of the vehicles. Estimation of the tire forces is essential in vehicle dynamics and control. This paper presents an observer-based scheme for estimation of the longitudinal tire force of electric vehicles in real time. The observer is based on a nonlinearity observer method. The pole-placement technique is used for determination of the observer gains. Simulation results demonstrate that the observer is able to estimate the tire force successfully. The experiments are implemented on a single-wheel electric vehicle test rig. The test rig comprises an electric motor driven wheel and a free-rolling drum simulating vehicle-on-road situations. Experimental results confirm the effectiveness of the present scheme.
\end{abstract}

Keywords: Estimation; Nonlinearity Observer; Tire Force; Traction Control; Electric Vehicles

\section{Introduction}

Electric vehicles (EVs) have become very attractive for replacing conventional internal combustion engine vehicles because of environmental and energy problems [1]. The research and development of EVs and hybrid EVs have been investigated on various topics such as, for example, propulsion systems [2], power converters [3], and motion control [4].

Traction control plays an important role in vehicle motion control because it increases drive efficiency, safety, and stability. Tire forces are essential in traction control. They are the vehicular propulsive forces produced by friction between the rolling wheel and the road surface. The characteristic of the friction between the wheel and the road surface is very nonlinear. It mainly depends on the wheel slip and the tire/road surface condition. In [5], an approach to estimate the tire-road friction during normal drive is presented. The approach is based on a Kalman filter to give estimates of the slip-slope. In [6], an on-line least-squares method is used to estimate the parameters concerned with a friction force margin. The effect of the estimation is evaluated by applying the method to the breaking control. A slip-based method to estimate the maximum available tire-road friction during breaking is developed in [7]. The method is based on the hypothesis that the slope of the slip curve at the low-slip region during normal driving can indicate the maximum friction coefficient. In [8], vehicle-dynamics-based methods for tire-road friction coefficients estimation are reviewed. The methods include slip-slope-based, lateralehicle-dynamics-based, and an EKF-based estimation methods. In [9], three different observers are developed for the estimation of slip ratios and longitudinal tire forces. The observers include one that utilizes engine torque, break torque, and GPS measurements, one that utilizes torque measurements and an accelerometer, and one that utilizes GPS measurements and an accelerometer.

This paper presents an observer-based scheme for estimation of the longitudinal tire force of EVs using a nonlinearity observer. Simulation and experimental studies are used to illustrate the effectiveness of the present scheme. A single-wheel test rig is used as an experimental test bench.

\section{Nonlinearity Observer}

The nonlinearity observer developed in $[10,11]$ is reviewed in this section. Consider the following nonlinear system

$$
\begin{aligned}
\dot{\mathbf{x}}(t) & =\mathbf{A x}(t)+\mathbf{N} \mathbf{f}(\mathbf{x}(t), t)+\mathbf{B u}(t) \\
\mathbf{y}(t) & =\mathbf{C x}(t)
\end{aligned}
$$

where $\mathbf{x}, \mathbf{u}$, and $\mathbf{y}$ are, respectively, the state vector, the control vector, and the output vector, respectively. $\mathbf{A}, \mathbf{B}, \mathbf{C}$ and $\mathbf{N}$ are, respectively, the system matrix, the control input matrix, the output matrix, and the 
nonlinearity matric. $\mathbf{f}(\cdot)$ is an unknown nonlinear function, which is estimated by the observer.

The fundamental idea of the observer is to approximate $\mathbf{f}(\cdot)$ by a fictitious system

$$
\begin{aligned}
& \mathbf{f}(\mathbf{x}(t), t) \approx \mathbf{H v}(t) \\
& \dot{\mathbf{v}}(t)=\mathbf{V} \mathbf{v}(t)
\end{aligned}
$$

By substituting Equation (2) into Equation (1), the observer can be chosen as

$$
\left[\begin{array}{c}
\dot{\hat{\mathbf{x}}} \\
\dot{\hat{\mathbf{v}}}
\end{array}\right]=\left[\begin{array}{cc}
\mathbf{A} & \mathbf{N H} \\
\mathbf{0} & \mathbf{V}
\end{array}\right]\left[\begin{array}{c}
\hat{\mathbf{x}} \\
\dot{\hat{\mathbf{v}}}
\end{array}\right]+\left[\begin{array}{c}
\mathbf{B} \\
\mathbf{0}
\end{array}\right] \mathbf{u}+\left[\begin{array}{l}
\mathbf{L}_{\mathbf{x}} \\
\mathbf{L}_{v}
\end{array}\right](\mathbf{y}-\mathbf{C} \hat{\mathbf{x}})
$$

where $\mathbf{L}_{\mathbf{x}}$ and $\mathbf{L}_{v}$ are the observer gain matrices that must be chosen such that the observer is asymptotically stable. In this paper, the pole-placement technique is used to obtain the observer gains. When $\mathbf{H}=\mathbf{I}$ and $\mathbf{V}=\mathbf{0}$ are chosen, the observer is reduced to a proportional-integral (PI) observer [11]

$$
\begin{aligned}
& \dot{\hat{\mathbf{x}}}=\mathbf{A} \hat{\mathbf{x}}+\mathbf{B u}+\mathbf{L}_{x}(\mathbf{y}-\mathbf{C} \hat{\mathbf{x}})+\mathbf{L}_{v} \int(\mathbf{y}-\mathbf{C} \hat{\mathbf{x}}) d t \\
& \hat{\mathbf{v}}=-\mathbf{L}_{v} \int(\mathbf{y}-\mathbf{C} \hat{\mathbf{x}}) d t
\end{aligned}
$$

and the estimated nonlinearity is given by

$$
\hat{\mathbf{f}}(t)=\hat{\mathbf{v}}(t) \text {. }
$$

\section{Single-Wheel Test Rig}

The single-wheel test rig used in the experiments is shown in Figure 1. It consists of a drum set, a wheel set and a measurement/control unit. The role of the drum set is to let the wheel to behave like rolling on the road. The diameter and width of the drum are approximately 1.0 and 0.3 meters, respective. The wheel set consists of a tire, a 4-inch-rim wheel, a brushed DC motor, and loading masses. The wheel is directly driven by the motor thought a rigid shaft. The measurement/control unit consists of a signal condition circuit and a PC computer. The computer is installed with a 12-bit analog/digital interface board. The reader is referred to [12] for more details of the test rig.

The mathematical model of the wheel set can be simply written as

$$
J_{1} \frac{d \omega_{1}}{d t}+\left(\frac{K_{T} K_{E}}{R}+C_{1}\right) \omega_{1}=\frac{K_{T}}{R} K_{0} V_{i n}-F_{\mu} r_{1}
$$

where $\omega_{1}$ is the rotational speed of the wheel, $V_{i n}$ is the input voltage applied to the motor, $F_{\mu}$ is the tire force, $r_{1}$ is the outer radius of the tire, $J_{1}$ is the total inertia of the rotating part including the wheel set, $C_{1}$ is the equivalent rotational damping constant, $K_{T}$ is the torque constant of the motor, $K_{E}$ is the back-EMF constant of the motor, $R$ is the resistance of the motor windings, and $K_{0}$ is the effective gain of the motor drive.

The mathematical model of the rotating drum can be expressed as

$$
J_{2} \frac{d \omega_{2}}{d t}+C_{2} \omega_{2}=F_{\mu} r_{2}
$$

where $\omega_{2}$ is the rotational speed of the drum, $r_{2}$ is the outer radius of the drum, $J_{2}$ is the total inertia of the drum, and $C_{2}$ is the equivalent rotational damping constant.

The tire force $F_{\mu}$ can be expressed as

$$
F_{\mu}=\mu(\lambda) N
$$

where $N$ is the normal force between the wheel and the drum, $\mu(\lambda)$ is the friction (or adhesion) coefficient, $\lambda$ is the driving slip ratio. The slip ratio is defined as

$$
\lambda=\frac{r_{1} \omega_{1}-v}{r_{1} \omega_{1}}, \quad \omega_{1} \neq 0
$$

where $v$ is the vehicle velocity. Here, since the motion of the vehicle velocity is simulated by the rotation of the drum, $v$ is substituted by the circumferential velocity of the drum, i.e.,

$$
v=r_{2} \omega_{2}
$$

The parameters of the model are determined by direct measurements and simulation tuning by comparing with real experimental data. The first set of the parameters are the mechanical parameters whose values are determined directly from measurements. The values are summarized in Table 1.

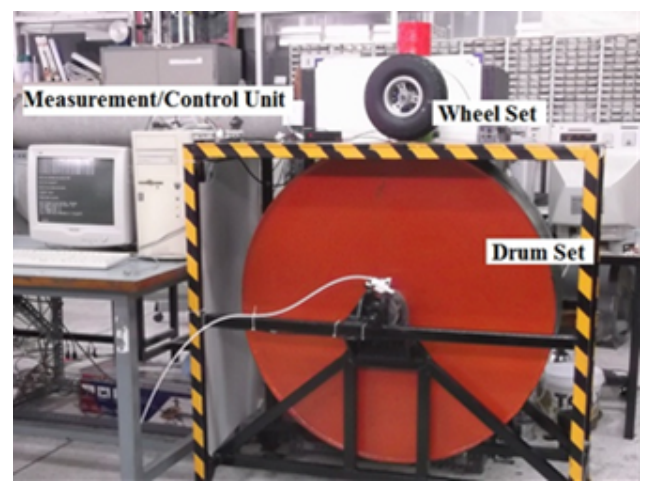

Figure 1. Photograph of the single-wheel test rig.

Table 1. Mechanical parameters of the test rig.

\begin{tabular}{cl}
\hline Symbol & \multicolumn{1}{c}{ Value } \\
\hline$J_{1}$ & $0.0098 \mathrm{~km} \mathrm{~m}^{2}$ \\
$J_{2}$ & $24.95 \mathrm{~km} \mathrm{~m}^{2}$ \\
$r_{1}$ & $0.13125 \mathrm{~m}$ \\
$r_{2}$ & $0.5 \mathrm{~m}$ \\
\hline
\end{tabular}


By substituting the values from Table 1 into Equations (6) and (7), it yields

$$
0.0098 \frac{d \omega_{1}}{d t}+a \omega_{1}=b V_{i n}-0.13125 F_{\mu}
$$

and

$$
24.95 \frac{d \omega_{2}}{d t}+C_{2} \omega_{2}=0.5 F_{\mu}
$$

where $a=\left(\frac{K_{T} K_{E}}{R}+C_{1}\right)$ and $b=\frac{K_{T}}{R} K_{0}$. The values of $a, b$, and $C_{2}$ are experimentally determined by measuring the step responses of the speeds of the wheel and the drum. Figure 2 shows an example of the step response. The initial velocities of the wheel and the drum are zero. There is also a step change of $V_{i n}$ at time $=$ $500 \mathrm{sec}$. After conducting several simulation tuning, the approximated values of the parameters are found to be $a=0.0392, b=0.4704$, and $C_{2} \approx 0.0$. In simulations, $\mu(\lambda)$ is set as

$$
\mu(\lambda)=\frac{2 \mu_{p} \lambda_{p} \lambda}{\lambda_{p}^{2}+\lambda^{2}}
$$

with $\lambda_{p}=0.3$ and $\mu_{p}=0.9$ are, respectively, the slip ratio and the friction coefficient when the maximum friction occurred.

Simulation results of the same operating condition done in the experiment are shown in Figure 3. The results confirm the validness of the simulation model. Note that there is high-slip behavior occurred in the first 50 sec. The simulation model is also able to detect this behavior, with some small errors.

\section{Observer Design}

To design an observer, it is assumed that the output is $\left[\begin{array}{ll}\omega_{1} & \omega_{2}\end{array}\right]^{T}$. Note that, in actual vehicles, $\omega_{1}$ can be easily obtained from an ABS speed sensor and $\omega_{2}$, which represents the vehicle velocity, can be computed from the velocity of a non-driven wheel or GPS measurements.

Equations (11) and (12) can be combined and written in the form of Equation (1) as

$$
\begin{aligned}
& \dot{\mathbf{x}}=\left[\begin{array}{cc}
-4 & 0 \\
0 & 0
\end{array}\right] \mathbf{x}+\left[\begin{array}{c}
-12.4 \\
0.2
\end{array}\right] F_{\mu}+\left[\begin{array}{c}
48 \\
0
\end{array}\right] \mathbf{u} \\
& \mathbf{y}=\left[\begin{array}{ll}
1 & 0 \\
0 & 1
\end{array}\right] \mathbf{x}
\end{aligned}
$$

where $\mathbf{x}^{T}=\left[\begin{array}{ll}\omega_{1} & \omega_{2}\end{array}\right]$. Here, $F_{\mu}$ is the nonlinearity estimated by the observer. From Equation (3) and let $\mathbf{H}=\mathbf{I}$ and $\mathbf{V}=\mathbf{0}$, the observer can be expressed as

$$
\dot{\hat{\mathbf{X}}}=\left[\begin{array}{ccc}
-4 & 0 & -12.4 \\
0 & 0 & 0.2 \\
0 & 0 & 0
\end{array}\right] \hat{\mathbf{X}}+\left[\begin{array}{c}
48 \\
0 \\
0
\end{array}\right] \mathbf{u}+\mathbf{L}\left(\mathbf{y}-\left[\begin{array}{ccc}
1 & 0 & 0 \\
0 & 1 & 0
\end{array}\right] \hat{\mathbf{X}}\right)(15)
$$

where $\hat{\mathbf{X}}^{T}=\left[\begin{array}{lll}\hat{\omega}_{1} & \hat{\omega}_{2} & \hat{F}_{\mu}\end{array}\right]$.

The pole-placement technique is used here to obtain the observer gain $\mathbf{L}$. The desired poles in continuous-time domain are simply chosen to be $-1,-2,-3$.

In implementations, the observer is implemented in a digital computer. The sampling period is set to be 0.5 second. Thus, the corresponding discrete-time desired poles are $0.6065,0.3679,0.2231$. Using the pole placement technique, it results the following observer gain

$$
\mathbf{L}=\left[\begin{array}{cc}
0.1612 & -0.1309 \\
-0.0021 & 0.7766 \\
-0.0859 & 0.0232
\end{array}\right]
$$

To verify the effectiveness of the observer, simulations are conducted in MATLAB/Simulink. The simulation results are shown in Figure 4. In Figure 4(a), the results show that the estimated values follow the true values very well. Figure 4(b) displays the responses for only the first $100 \mathrm{sec}$, where the system started from zero velocity. The results show that the observer estimates the tire force effectively even though the high slip occurred. The convergent time is less than $100 \mathrm{sec}$. The responses during the input voltage $V_{\text {in }}$ stepping up are shown in Figure 4(c). The estimates track the change effectively. In summary, the simulation results indicate that the observer is able to estimate the tire force successfully.
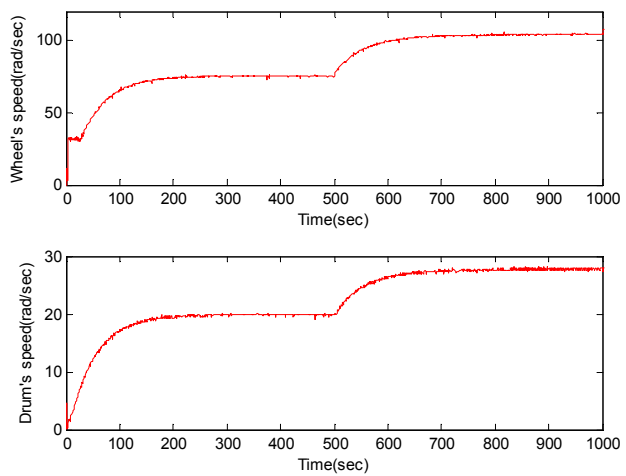

Figure 2. Step response of the experimental test rig.
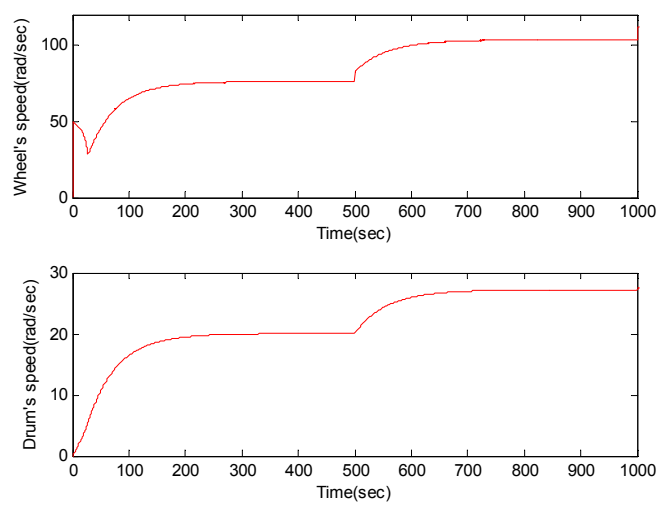

Figure 3. Step response of the simulation model. 

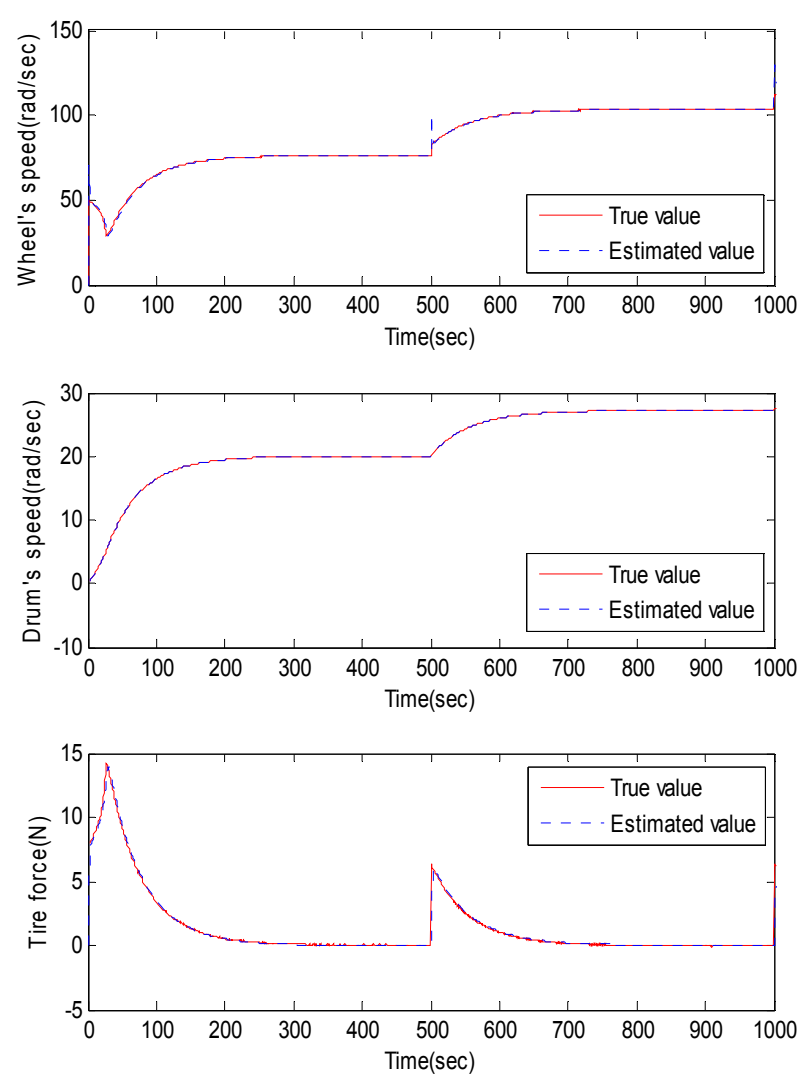

(a)
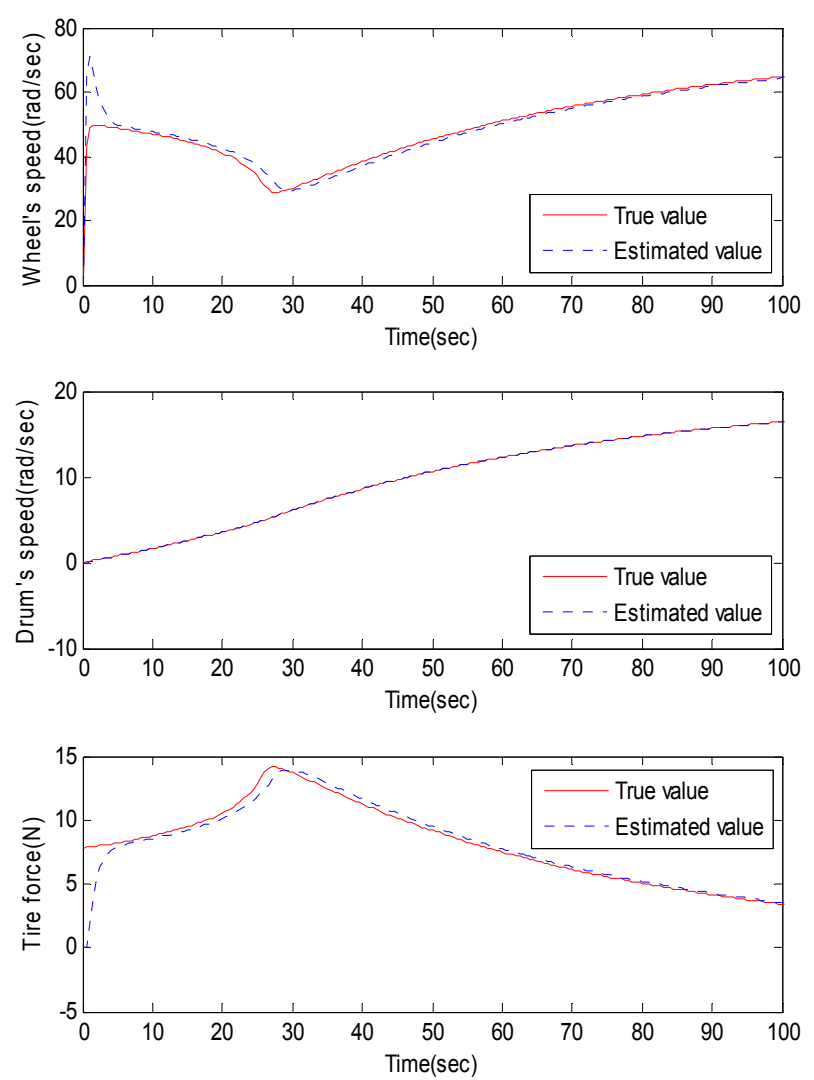

(b)
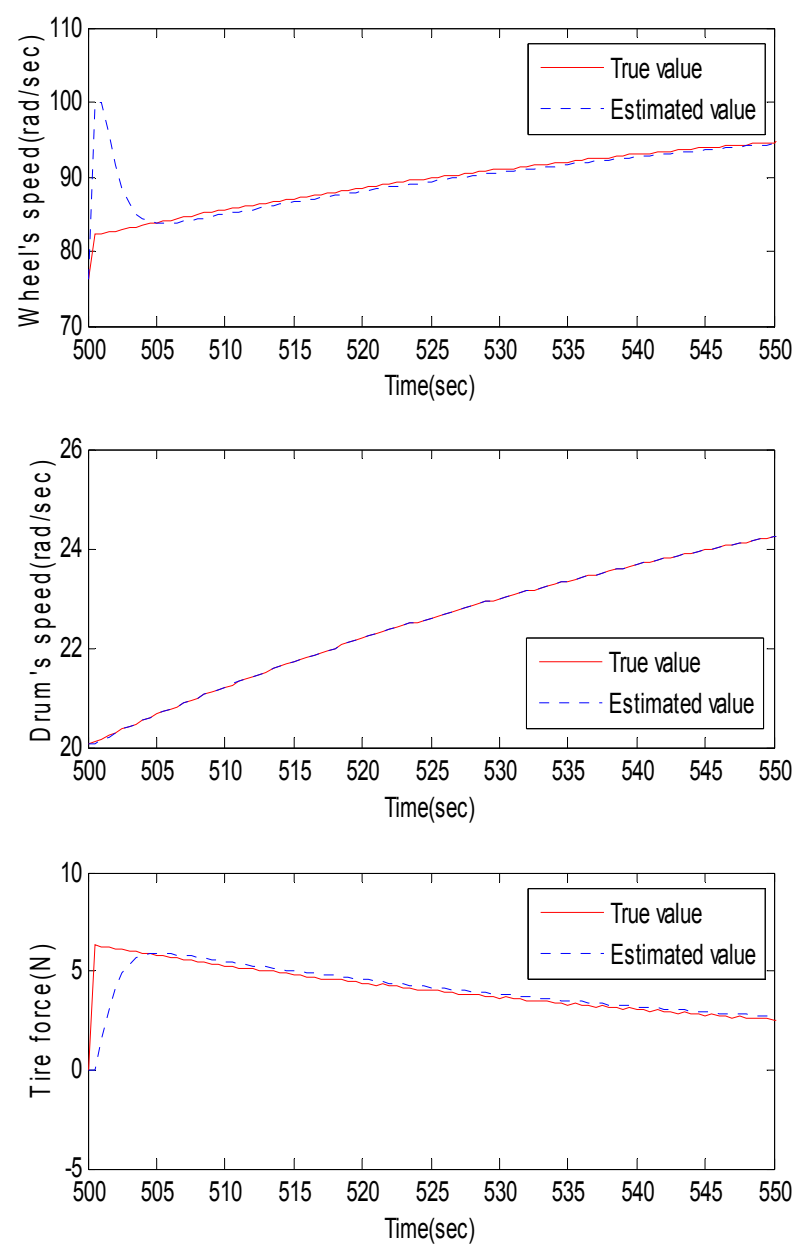

(c)

Figure 4. Simulation results: (a) time $=0-1000$ sec, (b) time $=0-100$ sec, (c) time $=\mathbf{5 0 0}-\mathbf{5 5 0}$ sec.

\section{Experimental Results}

The sampling rate of $0.5 \mathrm{sec}$ is used in all experiments. The same operation condition used in the simulations is repeated in the experiments. The experimental results are shown in Figure 5. They indicate that the observer is successfully implemented. Note that, in experiments, the true value of the tire force is unknown because it is not measurable. Since the experimental responses are very similar to those in the simulations, this implies that the estimates of the tire force is convincing.

\section{Conclusions}

Observer-based estimation of tire forces for road vehicles is studied in this paper. The observer is based on the nonlinearity observer developed in $[10,11]$. The poleplacement technique is used for determination of the observer gains. Both simulation studies and experiments are conducted in the paper. In the experiments, a single- wheel test rig is used as a testing platform. Both simulation and 

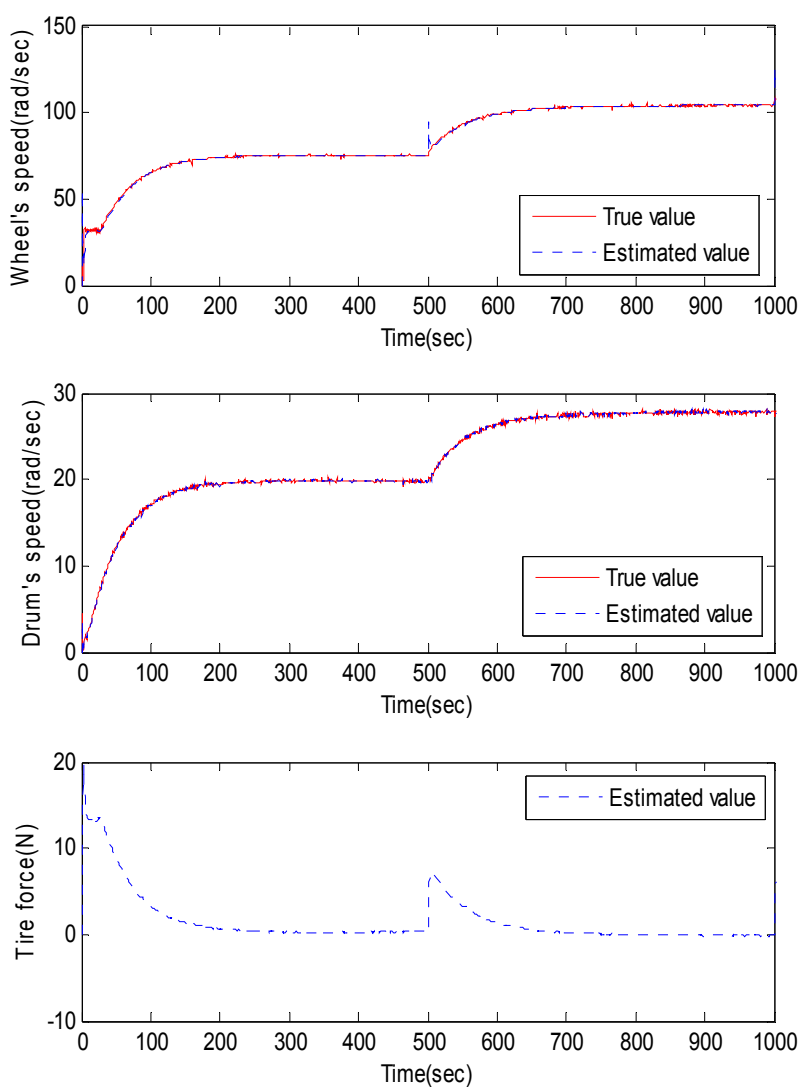

(a)
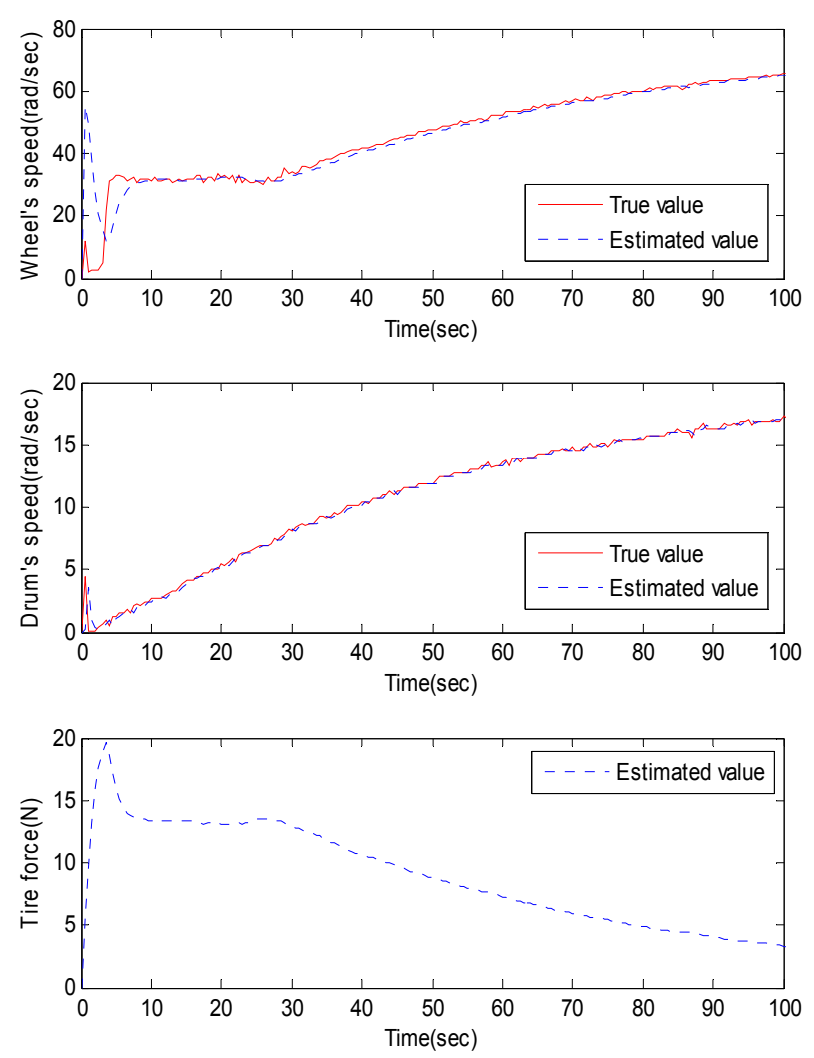

(b)
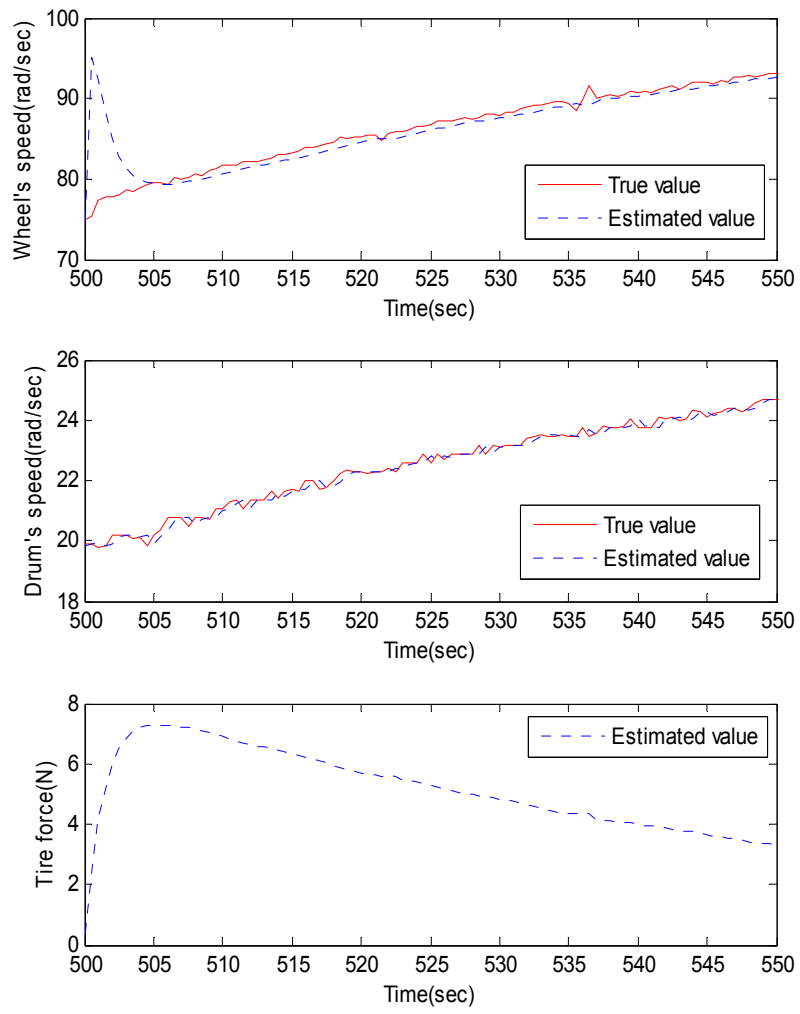

(c)

Figure 5. Experimental results: (a) time $=0-1000$ sec, (b) time $=0-100 \mathrm{sec},(\mathrm{c})$ time $=\mathbf{5 0 0}-\mathbf{5 5 0} \mathrm{sec}$.

experimental results show the effectiveness of the present observer.

\section{Acknowledgements}

The author gratefully acknowledges the support provided by Science and Technology Research Institute, King Mongkut's University of Technology North Bangkok .

\section{REFERENCES}

[1] C. C. Chan, "The State of the Art of Electric, Hybrid, and Fule Cel Vehicles," Proceedings of the IEEE, Vol. 95, No. 4, 2007, pp. 704-718. doi:10.1109/JPROC.2007.892489

[2] K. T. Chau, C. C. Chan and C. Liu, "Overview of Permanent-Magnet Brushless Drives for Electric and Hybrid Electric Vehicles," IEEE Transactions on Industrial Electronics, Vol. 55, No. 6, 2008, pp. 2246-2257. doi:10.1109/TIE.2008.918403

[3] M. J. Hoeijmakers and J. A. Ferreira, "The Electric Variable Transmission," IEEE Transactions on Industry Applications, Vol. 42, No. 4, 2006, pp. 1092-1093. doi:10.1109/TIA.2006.877736

[4] Y. Hori, "Future Vehicle Driven by Electricity and Control-Research on Four-Wheel-Motored 'UOT Electric March II'," IEEE Transactions on Industrial Electronics, Vol. 51, No. 5, 2004, pp. 954-962. 


\section{doi:10.1109/TIE.2004.834944}

[5] F. Gustafsson, "Slip-Based Tire-Road Friction Estimation," Automatica, Vol. 33, No. 6, 1997, pp. 1087-1099. doi:10.1016/S0005-1098(97)00003-4

[6] E. Ono, K. Asano, M. Sugai, S. Ito, M. Yamamoto, M. Sawada and Y. Yasui, "Estimation of Automotive Tire Force Chraracteristics Using Wheel Velocity," Control Engineering Practice, Vol. 11, 2003, pp. 1361-1370. doi:10.1016/S0967-0661(03)00073-X

[7] S. Müller, M. Uchanski and K. Hedrick, "Estimation of the Maximum Tire-Road Friction Coefficient," ASME J. Dynamic Systems, Measurement, and Control, Vol. 125, 2003, pp. 607-617. doi:10.1115/1.1636773

[8] R. Rajamani, D. Piyabongkarn, J. Y. Lew, K. Yi and G. Phanomchoeng, "Tire-Road Friction-Coefficient Estimation," IEEE Control Systems Magazine, Vol. 30, No. 4, 2010, pp. 54-69. doi:10.1109/MCS.2010.937006
[9] R. Rajamani, G. Phanomchoeng, D. Piyabongkarn and J. Y. Lew, "Algorithms for Real-Time Estimation of Individual Wheel Tire-Road Friction Coefficients," IEEE/ASME Trans. Mechatronics, Vol. 17, No. 6, 2012, pp.1183-1195.

[10] P. C. Müller, "Estimation and Compensation of Nonlinearities," Proceedings of the 1st Asian Control Conference, Tokyo, 1994, Vol. II, pp. 641-644.

[11] D. Söffker, T. J. Yu and P. C. Müller, "State Estimation of Dynamical Systems with Nonlinearities by Using ProPortional-Integral Observer," International Journal of Systems Science, Vol. 26, 1995, pp. 1571-1582. doi:10.1109/MCS.2010.937006

[12] A. Kraithaisri, S. Kuntanapreeda and S. Koetniyom, "Development of A Single-Wheel Test Rig for Traction Dynamics and Control of Electric Vehicles," Proceedings of the 2012 Hong Kong International Conference on Engineering and Applied Science, 2012, pp. 360-367. 